\title{
Investigating the Efficiency of Photothrombosis- associated Focal Cerebral Ischemia by Monitoring Histopathological Changes and Neurobehavioral Performance in a Model of Mouse
}

\section{Zahra Shabani \\ Tabriz University of Medical Sciences \\ Mehdi Farhoudi \\ Tabriz University of Medical Sciences \\ Reza Rahbarghazi \\ Tabriz University of Medical Sciences}

Mohammad Karimipour ( $\nabla$ karimipourm@yahoo.com )

Islamic Azad University, Tabriz Branch, Basic Sciences Faculty, Department of Physics. Neurosciences research center (NSRC), Tabriz University of Medical Sciences, Golgasht Street, Azadi Avenue, Tabriz, Iran. https://orcid.org/0000-0002-6975-4308

\section{Hossein Mehrad}

Tabriz Islamic Art University

\section{Research article}

Keywords: Stroke Model, Photothrombotic Stroke, Green Light, Rose Bengal dye, mNSS Test

Posted Date: August 31st, 2020

DOI: https://doi.org/10.21203/rs.3.rs-51657/v1

License: (c) (i) This work is licensed under a Creative Commons Attribution 4.0 International License. Read Full License 


\section{Abstract}

\section{Background}

Photothrombotic (PT) stroke model is a novel method to induce ischemic stroke in the target site using excitation of a photosensitive agent such as Rose Bengal dye after light illumination. Here, we performed a photothrombotic-induced brain ischemic model using green laser and photosensitive agent Rose Bengal (RB) and its efficiency evaluated through cellular, histological, and neurobehavioral approaches.

\section{Methods}

Mice were randomly allocated into RB; Laser irradiation; and RB + Laser irradiation groups. Mice were exposed to green laser at a wavelength of $532 \mathrm{~nm}$ and intensity $150 \mathrm{~mW}$ in a mouse model after injection of RB. Four circular polarizers were applied to control output beam intensity. The laser was illuminated on the target region under stereotactic surgery. Mice were euthanatized after 28 days. We assessed ischemic stroke outcomes using multiple approaches including macroscopic, microscopic (H\&E and Cresyl violet staining). The volume of lesion sites was calculated using unbiased stereological assay and Cavalier's principle. We performed double immunofluorescence staining for $\mathrm{NeuN}$ and $\mathrm{BrdU}$ on day 28. To assess the quality of ischemic stroke, neurological behavior, and functional deficits were done on days $1,7,14$, and 28 .

\section{Results}

Laser irradiation plus RB promoted pale ischemic changes after 7 days. Microscopic staining revealed neural tissue degeneration and demarcated necrotic site and neuronal injury plus prominent astrogliosis in the periphery of irradiated sites on day $28 . \mathrm{NeuN}^{+} / \mathrm{BrdU}^{+}$staining showed a significant number of proliferating cells in Laser irradiation plus RB group compared to the group $(p<0.05)$ while the percent of $\mathrm{NeuN}^{+}$cells reduced. Neurological behavior and functional deficits were detected in mice from Laser irradiation plus the RB group. No histological or functional deficits were detected in RB and Laser irradiation groups.

\section{Conclusion}

Our study revealed that the PT although increased the cell proliferating in the periphery of the lesion site, but due to undesirable microenvironment the neurogenesis decreased concomitantly with functional deficits. This method of PT introduces a reliable, reproducible, noninvasive, and accessible stroke model similar to human stroke condition.

\section{Background}

Stroke is the third cause of human mortality with long-lasting disability among the elderly population in the world [1]. One common cause of stroke is focal ischemic injury accounting for over $80 \%$ of all stroke cases occurs after the obstruction of blood vessels within certain brain areas, leading to neural tissue 
degeneration and neuronal death [2,3]. To elucidate underlying mechanisms after the promotion of ischemic stroke, researchers have exploited several animal models of focal brain ischemia [4]. The development of Ischemic Stroke is done via temporary or permanent ligation of middle cerebral artery occlusion (MCAO) [5]. Along with conventional approaches to induce ischemic stroke, thromboembolic ischemia is an alternative modality used via the injection of thrombin directly into the middle cerebral artery [6].

In recent decades, the acute photothrombotic (PT) stroke model has been developed for focal ischemic changes by local infusion photo-sensitive dye into the venous circulation $[7,8]$. Initially, Rosenblum and El-Sabban in 1977 introduced the PT model which later improved by Watson in 1985 [9]. Despite what's happening in human stroke, the PT model is considered as non-canonical ischemia because it does not block or break just one artery but damages more superficial vessels [10]. In this model, ischemic damage is induced within the targeted cortical region using photo-activation of previously infused light-sensitive dye [11]. The photo-activation of dye produces singlet oxygen radicals that impair the integrity of endothelial cells membrane [12]. The release of free radicals aggregates and adheres to red blood cells and platelets to the luminal surface of the vascular bed, causing thrombosis and ischemia in the brain cortex $[10,13]$.

PT is a competitive approach to other available methods of stroke induction. The lesion size is controllable to induce pre-determined infarct size by the operator without the need for surgical expertise $[14,15]$. In this method, the light source can be simply illuminated on the intact skull. Therefore, the PT model is a reproducible and non-invasive approach targeting cortical areas [10,16-18]. Besides, it has been shown that the mortality rate is low in animal models exposed to PT and enables us to maintain stroke animals for prolonged times [18]. Despite these advantages, there are some limitations to the promotion of stroke in animals by PT [19]. The intensity of vascular edema is massive due to the injury of endothelial cells soon after the induction of stroke by PT [20].

In this study, we introduced a simple and non-invasive PT approach to establish a mouse stroke model. The size and volume of focal stroke lesions were carefully analyzed by unbiased designed-base stereological analysis. To affirm the possible relationship between stroke lesions and functional performance, we did various neurological behavioral tests. In this regard, Neuropathological changes, including cell injury and glial scar and compensatory neurogenesis, were monitored using histological examination and immunofluorescence staining at the periphery of infarcted areas over 28 days.

\section{Methods}

\section{Ethical issue and considerations}

All experiments were conducted according to international principal guidelines and approved by a local ethics committee of Tabriz University of Medical Sciences (IR.TBZMED.VCR.REC.1397.239)

\section{Animals housing conditions and study design}


Thirty adult male 3-month-old mice, weighing (20-22 g), were purchased from laboratory animal husbandry of Tabriz University of Medical Sciences and maintained in the neuroscience laboratory a Research Center affiliated to Tabriz University of Medical Sciences. Before and after surgery, all animals were individually housed in separate standard propylene cages with a $12 \mathrm{~h}$ light-dark cycle in an airconditioned constant room temperature $\left(23 \pm 1^{\circ} \mathrm{C}\right)$. All animals had ad libitum free access to food and water. They were adapted to new housing conditions 14 days before the initiation of experimental procedures. [21]. The animals were randomly assigned into three groups (10 mice in each group) as follows; (I) Rose Bengal (RB) group: $150 \mu \mathrm{g} / \mathrm{kbw}$ RB was injected intraperitoneally (IP); (II) Laser irradiation group: mice were irradiated and (III) RB + Laser irradiation group: mice were received RB and exposed to laser irradiation. The design of study and experimental schedule was depicted in (Fig. 1).

\section{Protocol of PT-induced brain ischemia model}

\section{Systematic optimization and modification of the laser illumination}

Laser treatment at a wavelength of $532 \mathrm{~nm}$ and an intensity $150 \mathrm{~mW}$ was applied to induce PT-associate brain ischemia (Q-Switched Nd: YAG Laser). The system was equipped to a fan, heat sink, and a transistor-transistor logic-triggered power supply. In this study, we used 4 circular polarizers to concentrate the laser beam on the target sites (Fig. 1) [22]. The laser beams were passed through these circular polarizers with 90 degrees. To exclude hyperthermia during laser irradiation, a needle connected to thermocouple was located on the target part and temperature changes carefully recorded [13]. In this experiment, the temperature of the target zone reached $37^{\circ} \mathrm{C}$ after 10 minutes. To prevent the burning of the target zone, the time of radiation did not exceed more than 10 minutes and this time was used to PTinduced brain ischemia.

\section{General anesthesia}

In all groups, mice were anesthetized with the intraperitoneal injection of Ketamine hydrochloride ( $80 \mathrm{mg} / \mathrm{kg}$ ) and Xylazine hydrochloride $(5 \mathrm{mg} / \mathrm{kg}$ ) followed by monitoring body temperature throughout the experiment at $37^{\circ} \mathrm{C}$ using a rectal probe in conjunction with a thermocouple.

\section{Stereotaxic surgery}

Following anesthesia with the mixture of ketamine $(75 \mathrm{mg} / \mathrm{kg})$ and xylazine $(5 \mathrm{mg} / \mathrm{kg})$, mice in all groups were placed in a stereotactic device (Stoelting, USA) and the hair over the skull was shaved and skin was incised longitudinally $(1.0-1.5 \mathrm{~cm})$ using a sterile surgical blade. Then, the scalp was retracted to expose the skull surface. The periosteum was gently removed to clarify coronal and sagittal sutures. In this study, the following targeted site ( $1.1 \mathrm{~mm}$ anterior to the bregma and $2 \mathrm{~mm}$ lateral from the midline) was coordinated by the stereotaxic atlas of Paxinos and Watson. In the RB group, mice received $150 \mu \mathrm{g} / \mathrm{gbw}$ RB. 
To prepare RB solution, $150 \mathrm{mg} / \mathrm{ml} \mathrm{RB}$ dissolved in saline solution and sterilized through $0.2 \mu \mathrm{m}$-sized microfilters. In the RB + Laser irradiation group, an equal amount of RB dye was injected intraperitoneally. After diffusion and entrance of the RB into the bloodstream, the green laser was illuminated onto the skull surface ( $1.1 \mathrm{~mm}$ anterior to the bregma and $2 \mathrm{~mm}$ lateral from the midline). After the completion of laser irradiation, the light exposure was stopped and the wound area sutured. In the Laser irradiation group, mice were exposed to irradiation without administrating RB. After the completion of laser irradiation, mice were returned into their home cages under temperature-controlled conditions.

\section{BrdU incorporation assays}

To evaluate proliferation rate at the periphery of the stroke area, $10 \mathrm{mg} / \mathrm{ml} \mathrm{BrdU} \mathrm{(5-Bromo-2-Deoxyuridine;}$ Sigma) was dissolved in $0.9 \% \mathrm{NaCl}$ solution and then sterilized using by $0.22-\mu \mathrm{m}$ pore size microfilters (SPL). One day after stroke induction, all animals received IP injections of $\mathrm{BrdU}$ ( $50 \mathrm{mg} / \mathrm{kg}$ body weight) for 5 consecutive days.

\section{Measuring body weight and mortality rate post-PT}

Mice were weighed before the intervention and every day after stroke induction. Percent of body weight change was measured using the following formula: (body weight at each time after surgery-body weight before surgery)/body weight before surgery $\times 100 \%$ [23]. The rate of mortality was also assessed after ischemia induction according to the formula: (the number of death /the total number of mice in each group $\times 100 \%$ ) [24].

\section{Gross observation and histological examination The pattern of macroscopic damage after PT}

To this end, mice were humanely euthanized by the overdose administration of Ketamine and Xylazine. On days 1, 3, and 5 post-PT, we monitored a macroscopic feature of the affected area. We followed the existence of hyperemia, hemorrhagic and ischemic changes over 5 days.

\section{Tissue preparation, processing, and histological analysis}

To evaluate histopathological alterations, brain samples were taken on days 1, 3, and 5 after PT-induced brain ischemia. After deep sedation with Ketamine and Xylazine, mice were transcardially perfused with cold normal saline and $4 \%$ paraformaldehyde in $0.01 \mathrm{M}$ phosphate buffer $(\mathrm{pH}=7.4)$. Thereafter, the whole brain was dissected and post-fixed in the $4 \%$ paraformaldehyde solution at $4{ }^{\circ} \mathrm{C}$ overnight. After 24 hours, the tissues were cryoprotected in $30 \%$ sucrose in phosphate-buffered saline (PBS) at $4{ }^{\circ} \mathrm{C}$ overnight and sectioned coronally (5 $\mu \mathrm{m}$ thickness) into twelve serial coronal sections using a cryostat microtome $[25,26]$. The tissue sections were placed on gelatin-coated slides, rehydrated in descending series of alcohols $(100,90,80,70,50$ and 30), and distilled water. Then, samples were incubated in Hematoxylin for $5 \mathrm{~min}$ at room temperature. To exclude background staining, $0.3 \%$ acid alcohol was for $1-2$ seconds. After rinsing in tap water, slides were stained with Eosin solution for $2 \mathrm{~min}$ at room temperature followed 
by placing in increasing alcohols. Finally, the H\&E stained sections were observed and imaged under the light microscope [27].

\section{Assessment of infarct volume using unbiased stereological study}

To examine the effect of PT on cerebral tissue, lesion volume was measured on days 7, 14, and 28 after stroke induction based on Cavalier's principle. After perfusion fixation and brain sampling at respective time points, 12 serial coronal sections were obtained through the infarct region utilizing a systematic uniform random sampling design and a random start for stereological estimations. Each section was mounted on slides and stained with Cresyl violet solution to visualize the infarct lesion.

In this study, infarct volume was measured using stereology software (Computer Assisted Stereological Toolbox [CAST] software). For this purpose, the stereological probe was placed on the images of brain sections presented by a monitor. The total volume (TV) of the lesion was computed according to the following formula: Volume $\left(\mathrm{mm}^{3}\right)=\Sigma \mathrm{P} \times \mathrm{a} / \mathrm{p}\left(\mathrm{mm}^{2}\right) \times D(\mathrm{~mm})$; where $(\Sigma \mathrm{P})$ is the sum of the points falling on the lesion site in the brain section, $(a / p)$ is the area associated with each point at the level of the tissue section, and (D) is the distance between the sections [28, 29].

\section{Evaluation of neurogenesis by immunofluorescence staining}

To investigate the cell proliferation and neural differentiation at the periphery of stroke lesions, sections were labeled with anti-NeuN and anti-BrdU 28 days after ischemia induction. For this purpose, the antigens retrieval process was done by incubation of slides $10 \mathrm{mM}$ citrate buffer solution at $100^{\circ} \mathrm{C}$ for 15 min. Primary and secondary antibodies were diluted in TBS supplemented with $3 \%$ goat serum and 0.3\% TritonX-100. To permeabilize and block the samples, the sections were washed in TBS solution followed by incubation in 3\% goat serum and 0.3\% TritonX-100 for $30 \mathrm{~min}$. Thereafter, slides were incubated with anti-NeuN (Mouse anti-NeuN (Millipore, Chandlers Ford, Hants, United Kingdom; catalog No. MAB377; 1:100) and rat anti-BrdU (catalog No. OBT0030; 1:200; Accurate Chemical \& Scientific, Westbury, NY) antibodies at $4^{\circ} \mathrm{C}$ overnight. After washing twice in TBS, the mixture of secondary antibodies conjugated with Alexa Fluor 488 goat anti-mouse Alexa Fluor 488 catalog No. A-11001 [green; 1:200] and Alexa Fluor 568 goat anti-rat (catalog No. A-11077 [red; 1:200] (Invitrogen) were used.

For nuclear staining, 1 mg/ml 4', 6-Diamidino-2-phenylindole dihydrochloride (DAPI; Sigma-Aldrich) was used for 3 min. Finally, the sections were washed twice with TBS and mounted with glycerol buffer. A fluorescence microscope and digitally photographed software (Zeiss, Axiophot, Germany) were applied to visualize the positive cells. Besides, the percent of double-positive BrdU and NeuN cells were counted based on 100 BrdU-labeled cells per animal [29,30].

\section{Behavioral performance assessment}

\section{The cylinder test}


The cylinder test can assess the motor system deficit in the stroke model to evaluate spontaneous forelimb use in rodents [31]. To this end, the mice were placed in a transparent cylinder and forelimb movements observed. The mouse will naturally rear up on their hindlimbs and probe the vertical surface using the forelimbs and vibrissae. The blind examiner recorded the number of rights and left forelimbs placements on the cylinder's wall independently. According to scientific data, unilateral brain damage leads to an asymmetry in forelimb use during cylinder test [32,33]. This test is objective, easy to use, score, and requires no pre-training and help examiner to detect even mild neurological damages. Here, a total of 20 movements were recorded in each test and calculated according to the below formula;

The final score $=$ (non-impaired forelimb movement - impaired forelimb movement) $/$ (non-impaired forelimb movement + impaired forelimb movement + movement with both limbs).

\section{Modified neurological severity score (mNSS)}

mNSS test is one of the most common neurological scales usually utilized in animal stroke studies and is the potential to assess multiple impairments over 30-60 days period [34-37]. mNSS test was carried out to evaluate the behavioral performance before PT-induced brain ischemia after 1, 7, 14, and 28 days in a completely blinded manner. All indices such as motor and sensory function, balance, reflex, and general movements were measured on a scale of 18 ( $0=$ normal score; $18=$ maximal deficit score) (Table 1$)$. A score of 0 shows the lack of neurological deficit, whilst a score of 18 confirms the most severe injury. In this test, scores were arranged as follows; 0-6: mild neurological deficit; 7-12: moderate neurological impairment and 13-18: the severe neurological deficit. 
Table 1

Modified neurological severity score (mNSS): 1-6: mild injury; 7-12: moderate injury; 13-18: severe injury

\begin{tabular}{|c|c|c|}
\hline Items & Method & A detailed description of the items \\
\hline \multirow{7}{*}{$\begin{array}{l}\text { Motor tests } \\
(\text { Normal = 0, } \\
\text { Maximum = 6) }\end{array}$} & \multirow[t]{3}{*}{-Rising mouse by the tail } & -Flexion of the forelimb (1) \\
\hline & & -Flexion of the hindlimb (1) \\
\hline & & $\begin{array}{l}\text {-Head moved > } 10 \text { to vertical axis } \\
\text { within } 30 \mathrm{~S}(1)\end{array}$ \\
\hline & \multirow[t]{4}{*}{-Placing mouse on the floor } & -Normal walk (0) \\
\hline & & -Inability to walk straight (1) \\
\hline & & $\begin{array}{l}\text {-Circling toward the paretic side } \\
\text { (2) }\end{array}$ \\
\hline & & -Falls down to the paretic side (3) \\
\hline \multirow{2}{*}{$\begin{array}{l}\text { Sensory tests } \\
(\text { Normal = 0, } \\
\text { Maximum = 2) }\end{array}$} & -Visual and tactile test & $\begin{array}{l}\text {-Impaired visual and tactile } \\
\text { sensations (1) }\end{array}$ \\
\hline & $\begin{array}{l}\text {-Proprioceptive test by pushing the paw } \\
\text { against the table edge to stimulate the } \\
\text { muscles of the limb }\end{array}$ & $\begin{array}{l}\text {-Impaired proprioceptive } \\
\text { sensation (1) }\end{array}$ \\
\hline \multirow{7}{*}{$\begin{array}{l}\text { Beam balance } \\
\text { test } \\
(\text { Normal =0, } \\
\text { Maximum }=6)\end{array}$} & \multirow[t]{7}{*}{-Placing mice on a balance beam } & -Balance with steady posture (0) \\
\hline & & -Grasps side of the beam (1) \\
\hline & & $\begin{array}{l}\text {-Huges beam and } 1 \text { limb falls } \\
\text { down from the beam (2) }\end{array}$ \\
\hline & & $\begin{array}{l}\text {-Huges beam and } 2 \text { limbs fall } \\
\text { down -from beam or spines or } \\
\text { beam }(>40 \mathrm{~s})(3)\end{array}$ \\
\hline & & $\begin{array}{l}\text {-Attempts to balance on beam but } \\
\text { falls off }(>40 \mathrm{~s})(4)\end{array}$ \\
\hline & & $\begin{array}{l}\text {-Attempts to balance on beam but } \\
\text { falls off }(>20 \mathrm{~s})(5)\end{array}$ \\
\hline & & $\begin{array}{l}\text { Falls of, no attempt to balance, or } \\
\text { hang on the beam (6) }\end{array}$ \\
\hline $\begin{array}{l}\text { Reflex absence } \\
\text { and abnormal } \\
\text { movement }\end{array}$ & $\begin{array}{l}\text { - Pinna reflex: Touching the auditory } \\
\text { meatus and detecting whether the mouse } \\
\text { shakes his head }\end{array}$ & $\begin{array}{l}\text { Normal response: head shook } \\
\text { when the auditory meatus is } \\
\text { touched (1) }\end{array}$ \\
\hline \multirow[t]{3}{*}{$\begin{array}{l}(\text { Normal = 0, } \\
\text { Maximum = 4) }\end{array}$} & $\begin{array}{l}\text { - Cornea reflex: using the cotton to test the } \\
\text { blinking of mice }\end{array}$ & $\begin{array}{l}\text { Normal response: eye blink when } \\
\text { the cornea is touched with cotton } \\
\text { (1) }\end{array}$ \\
\hline & $\begin{array}{l}\text {-Startle reflex: playing a sudden loud noise } \\
\text { to test the motor response of mice }\end{array}$ & \multirow{2}{*}{$\begin{array}{l}\text { Normal response: the mouse } \\
\text { shows motor reaction to a brief } \\
\text { noise (1) }\end{array}$} \\
\hline & -Seizures, myoclonus, myodystonia & \\
\hline
\end{tabular}




\section{Motor function examination}

To test motor function, the mice were suspended by their tail to evaluate the forelimbs and hind limbs flexion and head movements in the vertical axis [33]. Under suspension conditions, rodents will extend their forelimbs toward the ground. After the promotion of stroke, mice will flex the contralateral forelimb and rotate the body towards the contralateral side of damage [38]. Besides, animals were positioned on a flat surface to analyze their gait. The detailed scoring of this function is described in Table 1.

\section{Sensory function examination}

The sensory test comprises the evaluation of visual, tactile, and proprioception $[33,35,36]$. The tactile function of mice was evaluated by touching the mice's body and limbs with cotton and the visual function was tested using a colorful subject and evaluating the response of mice to these stimulators. A proprioception test was assessed by pushing all four paws against the table edge or a narrow bar to stimulate the muscles of limbs. When the proprioceptive sensation is impaired, the mouse does not show appropriate muscle strength to paw the edge of the table or the bar with forelimbs or hindlimbs. The detailed scoring of this function is described in Table 1.

\section{Test of balance}

To evaluate animal balance, the mice were placed on a slim wooden beam that was $100 \mathrm{~cm}$ elevated from the ground [35, 39]. Maximal testing time was $60 \mathrm{~s}$ and the blinded examiner analyzed the position and walking of the mouse on the beam and counted the number of limbs that fell from the beam and the time before the mice fell off the beam. The detailed scoring of this function is summarized in Table 1.

\section{Reflexes tests}

The scored reflexes such as the pinna, corneal, and startle reflexes $[23,40,41]$. The pinna reflex was assessed by touching the auditory meatus and the detection of head shaking. To test corneal reflex, the cornea was stimulated slowly using the cotton or paper and the blinking of mice was assessed. A sudden loud noise was played to examine the startle reflex and the examiner recorded a motor reaction of mice to the noise. The presence of seizures, myoclonus, and myodystonia was evaluated [23]. The detailed scoring of this function is described in Table 1.

\section{Statistical Analysis}

Data were expressed in mean \pm SD and analyzed using Graph Pad Prism. One-Way ANOVA and posthoc Tukey tests were used to study group differences. We used the Kaplan-Meier method to analyze the mortality rate. Statistical significance was set at $p<0.05$. All studies were conducted blindly. 


\section{Results}

\section{PT-induced brain ischemia reduced body weight}

We monitored body weight changes in mice at $1,3,7$, and 14 and 28 days after PT-induced brain ischemia (Fig. 2A). Data showed that the bodyweight of mice was decreased quickly during the first 3 days after stroke induction and reached maximum levels on day $7(p<0.05 ;$ Fig. $2 A)$. We noticed that the bodyweight increased slowly after day 7 and an uptrend pattern observed from 14 to 28 days. However, the changes were statistically significant compared to the RB and Laser irradiation groups $(p<0.05$; Fig. 2A). The change in body weight in the same period (from 1 to 28 days) did not differ between RB and Laser irradiation $(p>0.05)$. These data showed that the induction of stroke via the PT method could decrease total body weight in the model mouse.

\section{PT-induced brain ischemia protocol did not cause mortality rate}

In the most common protocols for the induction of experimental stroke mainly MCAO, a high mortality rate is common in the rat and mouse models and the volume and intensity of brain injury are out of control [18]. We generated the Kaplan-Meier survival curve to measure the survival rate in mice after PTinduced stroke induction. Data analysis showed statistically non-significant differences in total mortality rate in RB + Laser irradiation group compared to the RB and Laser irradiation groups ( $p>0.05$; Fig. $2 B$ ). Interestingly, we recorded total mortality of $5 \%$ in $\mathrm{RB}+$ Laser irradiation group while no casualties were in the groups RB and Laser irradiation. All mortalities occurred during the early 3 days after induction of PTderived ischemia. Therefore, one could hypothesize that early-stage monitoring of mice is critical to calculating the whole mortality rate after the application of PT-induced brain ischemia.

\section{Macroscopic observation revealed neural tissue degeneration and ischemia after PT}

To confirm the promotion of ischemic changes, the skull was excised sagitally and scalp retracted to take the brain on days 1,3 , and 5 . Macroscopic observation revealed that simultaneous application of Laser irradiation and RB generated areas with a red-colored appearance (with an approximate radius of $2.5 \mathrm{~mm}$ ) after one day (Fig. 3A). The irradiated areas had delimited boundaries with magnificent hyperemia visible appearance by the unaided eye (Fig. 3A). On day 3 , we found ecchymosis vasodilation which became pale by the progression of time (day 5), confirming necrotic changes. H\& E staining revealed the necrotic lesions with well-delimited borders from peripheral areas after PT application on day 5 (Fig. 3A). No macroscopically-induced changes were shown in RB and Laser irradiation groups (Fig. 3A). No abnormality was detected on the contralateral hemisphere of experimental animals.

\section{Cresyl violet staining showed lesion volume alteration and neural cell injury}


Cresyl violet staining was used to evaluate infarct volume changes and possible neural injury on days 7 , 14, 28 following PT induction (Fig. 3B). A designed-based unbiased stereological study was used to estimate the lesion volume at respective time points. At low magnification, the ischemic core was restricted to the sensorimotor cortex and did not reach the subcortical region (Fig. 3B). The contralateral hemisphere in each experimental animal had no abnormality. In the ischemic group, the cresyl violet staining of brain sections revealed massive tissue degeneration at days 7 , leading to the formation of the infarct cavity in the irradiated areas (Fig. 3B). Histopathological examination showed local dense accumulation of active astrocytes in the periphery of lesion sites with elongated and spindle-like fibroblast appearance. These cells tended to form scar gliosis by time. Monitoring the neural cells at the periphery of irradiated sites showed pathological changes evident with chromatolysis phenomenon including cell soma swelling, and round-form appearance with eccentric nuclei, contributing to neural cell death and degeneration. No histological changes were found in the brain of mice received RB or Laser irradiation alone. According to our data, these values were initiated at the periphery of lesion sites on day 7 and intensified by time. The stereological analysis showed that the infarct volume decreased with the progression of time and became smaller at day 28 compared to days 7 and 14 ( $p<0.05$; Fig. $3 C$ ), suggesting spontaneous compensatory host tissue reaction to ischemic changes over time.

\section{Laser irradiation plus RB promoted cell proliferation and decreased neuronal differentiation}

We performed immunofluorescence imaging to monitor the rate of proliferation and neuronal phenotype in the peri-infarct area after laser irradiation (Fig. 4A-C). Data showed that the number of $\mathrm{BrdU}^{+}$cells was increased in the peri-infarct cortex of the RB + Laser irradiation group at day 28 post-ischemia induction compared to the RB and laser irradiation groups ( $p<0.05$; Fig. 4). No significant differences were found in the number of $\mathrm{BrdU}^{+}$cells between the RB and Laser irradiation groups ( $p>0.05$; Fig. 4). Double immunofluorescence staining of cells $\left(\mathrm{NeuN}^{+} / \mathrm{BrdU}^{+}\right)$was used to address this issue whether proliferating cells could commit to neural phenotype. We found that near $30 \%$ of $\mathrm{BrdU}^{+}$cells could differentiate into neural cells in both RB and Laser irradiation groups whilst this value was decreased significantly (below 20\%) in RB + Laser irradiation group compared to RB and Laser irradiation groups (p $<0.05$; Fig. 4). Taken together, the promotion of ischemic changes could induce proliferation of cells at the periphery of the lesion site and a small fraction of proliferating cells could orient to neural lineage.

\section{PT-associated brain ischemia-induced neurological deficit and behavior disabilities}

The infarct volume is an accurate hallmark of stroke, neurological deficit analysis by behavioral tests can be another meaningful parameter for detecting brain ischemia. Before ischemia, mice exhibited no neurologic deficits when evaluated by cylinder and mNSS tests examination. Conversely, all mice showed a significant decline in neurologic function after stroke induction and an increase in neurologic deficit score. 


\subsubsection{The cylinder test evaluation}

Data analysis showed that PT-induced mice used their contralateral paw dramatically much less than the control groups on days $1,7,14$, and 28 after ischemia but this deficit gradually reduced by proceeding time (Fig. 5a-d).

\section{The mNSS test evaluation}

The PT infarcts caused mild to moderate, long term sensorimotor impairment, well indicated by the mNSS test on days 1, 7, 14, and 28 after PT (Fig. 5e-r). In our model, neurological deficits were evident 1 hour after PT induction and were markedly higher in days 1-7 but gradually decreased over time.

\section{Motor function evaluation}

Forelimb flexion in all mice was observed when the mice raised by the tail, but the movement of the hindlimb and head control were not impaired in stroke mice (Fig. 5e-g). Further, the mice were unable to walk straight and sometimes, fell on the contralateral side. However, the mice in the RB and laser irradiation received groups did not exhibit any motor function impairment when assayed by mNSS.

\section{Sensory function evaluation}

Due to the interruption in controlling forelimb movement unilaterally, the mice revealed proprioception sensory deficit when the paws were pushed against the table edge or narrow bar to stimulate the muscles of limb and they were not able to paw the table edge or bar with front paws (Fig. 5h-j). We did not show any tactile and visual sensory impairment in PT induced mice and animals appropriately responded to the touching with cotton. However, in the RB and Laser irradiation groups, we did not find any of the mentioned sensory disruptions.

\section{Balance control evaluation}

Disability in controlling balance on a wooden beam was found in mice undergone PT stroke (Fig. 5k-n). The ischemic mice were not able to balance on the beam with steady posture and either hugged the beam or attempted to balance on the beam but fell out after 20 seconds. However, the mice in the RB and Laser irradiation groups did not exhibit any balance disruption and path through the wooden beam successfully.

\section{Reflex examination evaluation}

The cornea reflex was impaired in the mice after ischemia induction but pinna and startle reflexes were normal (Fig. 5o-q). Conversely, control mice that received RB alone or irradiation alone did not exhibit abnormalities in reflexes (Fig. 5o-q). Seizures, myoclonus, myodystonia were not observed in all groups.

\section{Discussion}


Light irradiation after the injection of photosensitive dyes is an approach to develop an experimental stroke model in laboratory animal models $[10,11]$. The generation of reactive oxygen species by photosensitive dyes triggers clotting cascades, leading to ischemia-induced pathological changes in the targeted region which apply to the human counterpart observed in the clinical setting [18]. In this research, a green laser from a filtered xenon arc lamp was selected to activate RB at the wavelength of $562 \mathrm{~nm}$ and $150 \mathrm{~mW}$ using cold light optic fibers. This approach enables us to expedite irradiation easily at a significantly low price [42]. Also, four circular polarizers were used to control output beam intensity and successfully activate light-sensitive dye without heat generation and unwanted burn in the target zone. The molecules of a polarizing filter were aligned in the same direction. Vibrating molecules could absorb the light waves that have the same orientation as the filter; therefore they decrease the intensity and scattering of the light passing through these filters [22]. In this experiment, the temperature of the target zone reached $37^{\circ} \mathrm{C}$ after 10 minutes. The time of radiation did not exceed more than 10 minutes and this time was used to PT-induced brain ischemia. Therefore, based on our experiment, it seems that the application of the polarized laser beam could be a safe and reliable method among the diversity of the PT stroke model. The lack of salvageable penumbra and high mortality rates are the main problems of other conventional stroke models [43]. In our previous experiment, we evaluated the histopathological changes from 1 to 7 days after PT induction using morphometric and stereological methods [44]. Here, in the present study, we decided to investigate whether this technique is reproducible or not and also, we would like to extend the follow up the focal cerebra ischemia outcomes on cerebral tissue architecture, particularly cellular behavior and neurobehavioral performance for a prolonged period (28 days) following to PT. Despite prominent weight alteration, the existence of insignificant causalities indicates the safety of the current model in the induction ischemic stroke in the model of mice. Histological examination showed the ischemic brain changes as early as 1 day indicated by disseminated hyperemia after PT-induced brain ischemia protocol. The pattern of hyperemia turned into ecchymosis vasodilation on day 3 which further became pale by the progression of time after 5 days, confirming necrotic changes. The quantitative stereological analysis revealed the reduction of the infarct cavity volume, reaching minimum levels on day 28 . Since the level of neurological deficits and recovery after ischemia is deeply linked to both the infarct size and neurovascular structure of the peri-infarct area [45], therefore, it is not surprising that largest functional deficits were observed 1 to 7 days post-ischemic that reduced gradually over time and recovery initiated thereafter. Bright-field microscopic imaging showed bulk degeneration and sloughing of ischemic areas after PT-induced ischemia on day 7 , leading to the generation of vacant space. In contrast to ischemic volume changes, the number of active astrocytes was increased and exhibited in the periphery of lesion sites with elongated and spindle-like fibroblast appearance. These cells tended to form scar gliosis by time. Additionally, massive chromatolysis such as cell soma swelling, and round-form appearance with eccentric nuclei were seen in the periphery of irradiated areas, contributing to neural cell death and degeneration. These features were correlated with the dynamic activity of astrocytes and the characteristic hallmarks of pro-inflammatory responses after the occurrence of ischemic changes [46]. In the present study, the number of BrdU labeled cells significantly increased around the infarct cavity on day 28 compared to RB and laser irradiation groups, indicating increased cell proliferation and migration at the periphery of ischemic areas. We also performed double $\mathrm{NeuN}^{+} / \mathrm{Brdu}^{+}$ 
staining to measure the rate of neurogenesis at the periphery of lesion sites. In this regard, we calculated the $\mathrm{NeuN}^{+} / \mathrm{BrdU}^{+}$ratio. Despite enhanced proliferation rate at the periphery of lesion sites in the $\mathrm{RB}+$ Laser irradiation group, our data showed that a small fraction of proliferating cells belong to neuronal lineage compared to the other groups. This data showed that astrogliosis is the main host tissue response in the injured brain. Theoretically, it has been demonstrated new neurons are produced under certain pathological conditions such as brain ischemia to regenerate the damaged region to improve the neurological functions [47]. Consistent with current data, some previous studies indicated endogenous neurogenesis occurred after stroke and other ischemic insults [48, 49]. Even neuronal differentiation was not completely stopped in our study but this capacity diminished significantly compared to the health condition. Abati et al indicated that subventricular zone (SVZ)- derived neural stem cells(NSCs) migrate to the damaged cortex after an injury, where a subpopulation will generate reactive astrocytes [50]. Faiz et al reported that SVZ-derived NSCs migrate to the stroke lesion after lesion and rapidly differentiate and give rise to a subpopulation of reactive astrocytes, suggesting a role for the SVZ in post-injury gliosis [51]. The lack of sufficient compensatory neurogenesis is due to the undesirable microenvironment, storm release of cytokines, and reduction of appropriate growth factors that coincided with increased differentiation towards astrocyte lineage [52].

\section{Conclusions}

According to the above-mentioned findings, the current study showed ischemic changes and behavioral deficits a few days after PT in the target areas. Besides, enhanced BrdU ${ }^{+}$proliferating cells around the lesion site suggest reactive astrogliosis and disoriented brain tissue remodeling. Taken together, the current approach is suitable to induce experimental stroke similar to mechanisms occurred in human in vivo condition.

\section{Abbreviations}

PT

Photothrombotic; RB:Rose Bengal; H\&E:Hematoxylin and Eosin; NeuN:Neuronal nuclei BrdU:Bromodeoxyuridine / 5-bromo-2'-deoxyuridine:mNSS:Modified neurological severity score; MCAO:Middle Cerebral Artery Occlusion; IP:Intraperitoneal; CAST:Computer Assisted Stereological Toolbox; TV:Total Volume; a/p:Area per Point; D:Distance; IF:Immunofluorescence; DAPI:4', 6-Diamidino-2phenylindole dihydrochloride; SVZ:Subventricular zone; NSCs:Neural Stem Cells

\section{Declarations}

Ethics approval and consent to participate The study was approved by the local animal ethics committee Local Ethics Committee, Tabriz University of Medical Sciences (IR.TBZMED.VCR.REC.1397.239). All animals were purchased from Laboratory Animal Husbandry of Tabriz University of Medical Sciences.

Consent for publication None applicable 
Availability of data and material The datasets used and/or analyzed during the current study are available from the corresponding author on reasonable request.

Competing interests The authors declare that they have no competing interests.

Funding This work was supported by grants (60232) from the Vice Chancellor for Research and Neurosciences Research Center (NSRC) of Tabriz University of Medical Sciences. This funding organization itself played no role in study design; in data collection, analysis, or interpretation; or in writing the manuscript.

Authors' contributions Z.S., M.F., and R.R.: Methodology, Investigation, and Data Curation; H.M: Laser irradiation; M. K: Conceptualization, Supervision, Funding acquisition. All authors have read and approved the manuscript.

Acknowledgment Authors wish to thank the personnel of Neurosciences Research Center for guidance and help

\section{References}

1. Kowalczyk K, Gasecki D, Kwarciany M, Jablonski B, Narkiewicz K, Karaszewski B. Short-term Functional Outcome After Ischemic Stroke Is Related To Blood Pressure Variability. J Hypertens. 2018;36:e126-e7.

2. Nita H: Ischemic Stroke Therapy. In.: Google Patents; 2012.

3. Li W, Qu Z, Prakash R, Chung C, Ma H, Hoda MN, et al. Comparative analysis of the neurovascular injury and functional outcomes in experimental stroke models in diabetic Goto-Kakizaki rats. Brain research. 2013;1541:106-14.

4. McCabe C, Arroja MM, Reid E, Macrae IM. Animal models of ischaemic stroke and characterisation of the ischaemic penumbra. Neuropharmacology. 2018;134:169-77.

5. McBride DW, Zhang JH. Precision stroke animal models: the permanent MCAO model should be the primary model, not transient MCAO. Translational stroke research. 2017;8 5:397-404.

6. Drieu A, Buendia I, Levard D, Hélie P, Brodin C, Vivien D, et al. Immune responses and antiinflammatory strategies in a clinically relevant model of thromboembolic ischemic stroke with reperfusion. Translational Stroke Research. 2020;11:3:481-95.

7. Gorlamandala N, Parmar J, Craig AJ, Power JM, Moorhouse AJ, Krishnan AV, et al. Focal ischaemic infarcts expand faster in cerebellar cortex than cerebral cortex in a mouse photothrombotic stroke model. Translational stroke research. 2018;9 6:643-53.

8. Li H, Zhang N, Lin H-Y, Yu Y, Cai Q-Y, Ma L, et al. Histological, cellular and behavioral assessments of stroke outcomes after photothrombosis-induced ischemia in adult mice. BMC Neurosci. 2014;15(1):58.

9. Svoboda J. Model mozkové fokální korové ischémie a jeho parametrizace. 2015. 
10. Labat-gest $\mathrm{V}$, Tomasi S. Photothrombotic ischemia: a minimally invasive and reproducible photochemical cortical lesion model for mouse stroke studies. Journal of visualized experiments: JoVE. 2013; 76.

11. Shah AM, Ishizaka S, Cheng MY, Wang EH, Bautista AR, Levy S, et al. Optogenetic neuronal stimulation of the lateral cerebellar nucleus promotes persistent functional recovery after stroke. Scientific reports. 2017;7:46612.

12. Lunardi Baccetto S, Lehmann C. Microcirculatory Changes in Experimental Models of Stroke and CNS-Injury Induced Immunodepression. Int J Mol Sci. 2019;20 20:5184.

13. Alaverdashvili M, Paterson PG, Bradley MP. Laser system refinements to reduce variability in infarct size in the rat photothrombotic stroke model. J Neurosci Methods. 2015;247:58-66.

14. Krakauer JW, Carmichael ST, Corbett D, Wittenberg GF. Getting neurorehabilitation right: what can be learned from animal models? Neurorehabilitation and neural repair. 2012;26 8:923 - 31.

15. Murphy TH, Corbett D. Plasticity during stroke recovery: from synapse to behaviour. Nature reviews neuroscience. 2009;10 12:861.

16. Li H, Zhang N, Sun G, Ding S. Inhibition of the group I mGluRs reduces acute brain damage and improves long-term histological outcomes after photothrombosis-induced ischaemia. ASN neuro. 2013;5 3:AN20130002.

17. Wang T, Cui W, Xie Y, Zhang W, Ding S. Controlling the volume of the focal cerebral ischemic lesion through photothrombosis. Am J Biomed Sci. 2010;2:33-42.

18. Watson BD, Dietrich WD, Busto R, Wachtel MS, Ginsberg MD. Induction of reproducible brain infarction by photochemically initiated thrombosis. Annals of Neurology: Official Journal of the American Neurological Association the Child Neurology Society. 1985;17 5:497-504.

19. Bix GJ, Gowing EK, Clarkson AN. Perlecan domain V is neuroprotective and affords functional improvement in a photothrombotic stroke model in young and aged mice. Translational stroke research. 2013;4 5:515-23.

20. Carmichael ST. Rodent models of focal stroke: size, mechanism, and purpose. NeuroRx. 2005;2 3:396-409.

21. Karimipour M, Rahbarghazi R, Tayefi $H$, Shimia M, Ghanadian M, Mahmoudi J, et al. Quercetin promotes learning and memory performance concomitantly with neural stem/progenitor cell proliferation and neurogenesis in the adult rat dentate gyrus. Int J Dev Neurosci. 2019;74:18-26.

22. Ichihashi M, Majima W, Oki K: Circular polarizing filter and application thereof. In.: Google Patents; 2018.

23. Wang J, Yu L, Jiang C, Chen M, Ou C, Wang J. Bone marrow mononuclear cells exert long-term neuroprotection in a rat model of ischemic stroke by promoting arteriogenesis and angiogenesis. Brain, behavior, and immunity. 2013;34:56-66.

24. Fisker AV, Stage I, Philipsen HP. Use of etorphine-acepromazine and diprenorphine in reversible neuroleptanalgesia of rats. Laboratory animals. 1982;16(2):109-13. 
25. Iqbal G, Ahmed T. Co-exposure of metals and high fat diet causes aging like neuropathological changes in non-aged mice brain. Brain research bulletin. 2019;147:148-58.

26. Chen Z, Chopp M, Zacharek A, Li W, Venkat P, Wang F, et al. Brain-derived microparticles (BDMPs) contribute to neuroinflammation and Lactadherin reduces BDMP induced neuroinflammation and improves outcome after stroke. Front Immunol. 2019;10:2747.

27. Yu X, Wang X, Zeng S, Tuo X. Protective effects of primary neural stem cell treatment in ischemic stroke models. Experimental therapeutic medicine. 2018;16 3:2219-28.

28. Kheradpezhouh E, Miri R, Noorafshan A, Panjehshahin M-R, Mehrabani D. A new method of brain staining with triphenyltetrazolium chloride to determine the infarct size in rats. Journal of Applied Animal Research. 2008;33:2:149-52.

29. Esfandiary E, Karimipour M, Mardani M, Alaei H, Ghannadian M, Kazemi M, et al. Novel effects of Rosa damascena extract on memory and neurogenesis in a rat model of Alzheimer's disease. $J$ Neurosci Res. 2014;92(4):517-30. doi:10.1002/jnr.23319.

30. Nasrolahi A, Mahmoudi J, Karimipour M, Akbarzadeh A, Sadigh-Eteghad S, Salehi R, et al. Effect of cerebral dopamine neurotrophic factor on endogenous neural progenitor cell migration in a rat model of Parkinson's disease. EXCLI J. 2019;18:139.

31. Gharbawie OA, Whishaw PA, Whishaw IQ. The topography of three-dimensional exploration: a new quantification of vertical and horizontal exploration, postural support, and exploratory bouts in the cylinder test. Behav Brain Res. 2004;151(1-2):125-35.

32. Bland ST, Schallert T, Strong R, Aronowski J, Grotta JC. Early exclusive use of the affected forelimb after moderate transient focal ischemia in rats. Stroke. 2000;31 5:1144-52.

33. Markgraf CG, Green EJ, Hurwitz BE, Morikawa E, Dietrich WD, McCabe PM, et al. Sensorimotor and cognitive consequences of middle cerebral artery occlusion in rats. Brain research. 1992;575(2):23846.

34. Shehadah A, Chen J, Zacharek A, Cui Y, lon M, Roberts C, et al. Niaspan treatment induces neuroprotection after stroke. Neurobiol Dis. 2010;40(1):277-83.

35. Li Y, Chen J, Wang L, Lu M, Chopp M. Treatment of stroke in rat with intracarotid administration of marrow stromal cells. Neurology. 2001;56 12:1666-72.

36. Chen J, Zhang C, Jiang H, Li Y, Zhang L, Robin A, et al. Atorvastatin induction of VEGF and BDNF promotes brain plasticity after stroke in mice. Journal of cerebral blood flow Metabolism. 2005;25(2):281-90.

37. Zhang L, Schallert T, Zhang ZG, Jiang Q, Arniego P, Li Q, et al. A test for detecting long-term sensorimotor dysfunction in the mouse after focal cerebral ischemia. J Neurosci Methods. 2002;117(2):207-14.

38. Modo M, Stroemer R, Tang E, Veizovic T, Sowniski P, Hodges H. Neurological sequelae and long-term behavioural assessment of rats with transient middle cerebral artery occlusion. J Neurosci Methods. 2000;104 1:99-109. 
39. Luong TN, Carlisle HJ, Southwell A, Patterson PH. Assessment of motor balance and coordination in mice using the balance beam. JoVE (Journal of Visualized Experiments). 2011;49:e2376.

40. GERMANÒ AF, DIXON CE, d'AVELLA D, HAYES RL. TOMASELLO F. Behavioral deficits following experimental subarachnoid hemorrhage in the rat. $J$ Neurotrauma. 1994;11 3:345-53.

41. Chen Y, Lomnitski L, Michaelson D, Shohami E. Motor and cognitive deficits in apolipoprotein Edeficient mice after closed head injury. Neuroscience. 1997;80 4:1255-62.

42. Van Reempts J, Van Deuren B, Van de Ven M, Cornelissen F, Borgers M. Flunarizine reduces cerebral infarct size after photochemically induced thrombosis in spontaneously hypertensive rats. Stroke. 1987;18 6:1113-9.

43. Ingberg E, Dock H, Theodorsson E, Theodorsson A, Ström JO. Method parameters' impact on mortality and variability in mouse stroke experiments: a meta-analysis. Scientific reports. 2016;6:21086-. doi:10.1038/srep21086.

44. Shahi M, Abedelahi A, Mohammadnejad D, Rahbarghazi R, Rasta SH, Karimipour M. Exact location of sensorimotor cortex injury after photochemical modulation; evidence of stroke based on stereological and morphometric studies in mice. Lasers in medical science. 2020. doi:10.1007/s10103-020-03017-y.

45. Mărgăritescu O, Mogoantă L, Pirici I, Pirici D, Cernea D, Mărgăritescu C. Histopathological changes in acute ischemic stroke. Rom J Morphol Embryol. 2009;50:3:327-39.

46. Haupt C, Witte OW, Frahm C. Up-regulation of Connexin43 in the glial scar following photothrombotic ischemic injury. Mol Cell Neurosci. 2007;35 1:89-99.

47. Palma-Tortosa S, García-Culebras A, Moraga A, Hurtado O, Perez-Ruiz A, Durán-Laforet V, et al. Specific features of SVZ neurogenesis after cortical ischemia: a longitudinal study. Sci Rep. 2017;7 $1: 1-12$.

48. Li H, Zhang N, Lin H-Y, Yu Y, Cai Q-Y, Ma L, et al. Histological, cellular and behavioral assessments of stroke outcomes after photothrombosis-induced ischemia in adult mice. BMC Neurosci. 2014;15(1):1-13.

49. Kokaia Z, Lindvall O. Neurogenesis after ischaemic brain insults. Curr Opin Neurobiol. 2003;13:1:127-32.

50. Abati E, Bresolin N, Comi GP, Corti S. Preconditioning and cellular engineering to increase the survival of transplanted neural stem cells for motor neuron disease therapy. Mol Neurobiol. 2019;56 5:335667.

51. Faiz M, Sachewsky N, Gascón S, Bang KA, Morshead CM, Nagy A. Adult neural stem cells from the subventricular zone give rise to reactive astrocytes in the cortex after stroke. Cell stem cell. 2015;17 5:624-34.

52. Dibajnia P, Morshead CM. Role of neural precursor cells in promoting repair following stroke. Acta Pharmacol Sin. 2013;34 1:78-90.

\section{Figures}




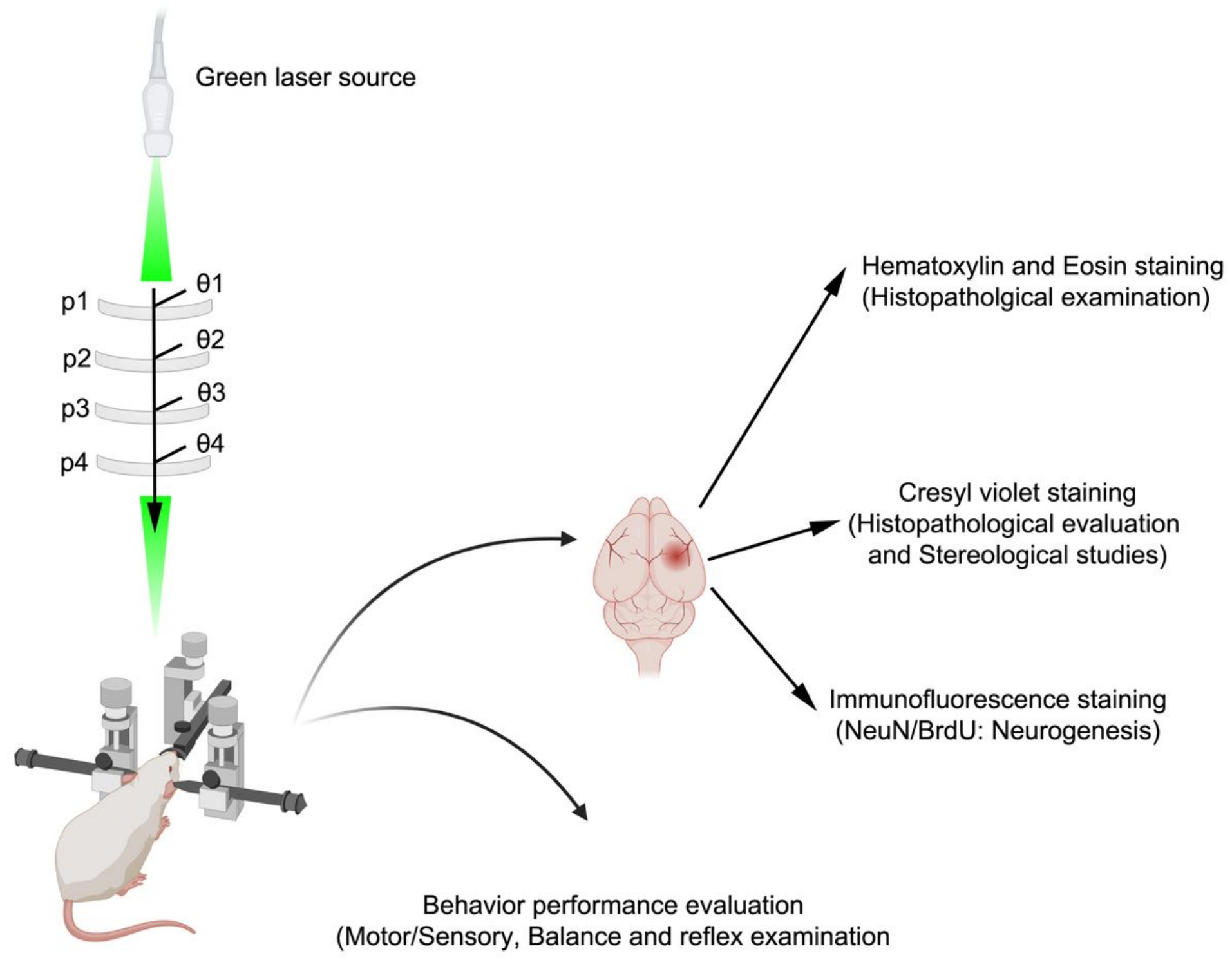

Figure 1

Schematic representation of PT-induced ischemic stroke and relevant analyses. PT-induced ischemic stroke was done under stereotaxic surgery by illuminating polarized green laser to activate RB dye using polarizers $\left(\mathrm{P} 1-\mathrm{P} 4 ; \theta=90^{\circ}\right)$. The figure was designed using web-based tool BioRender. 

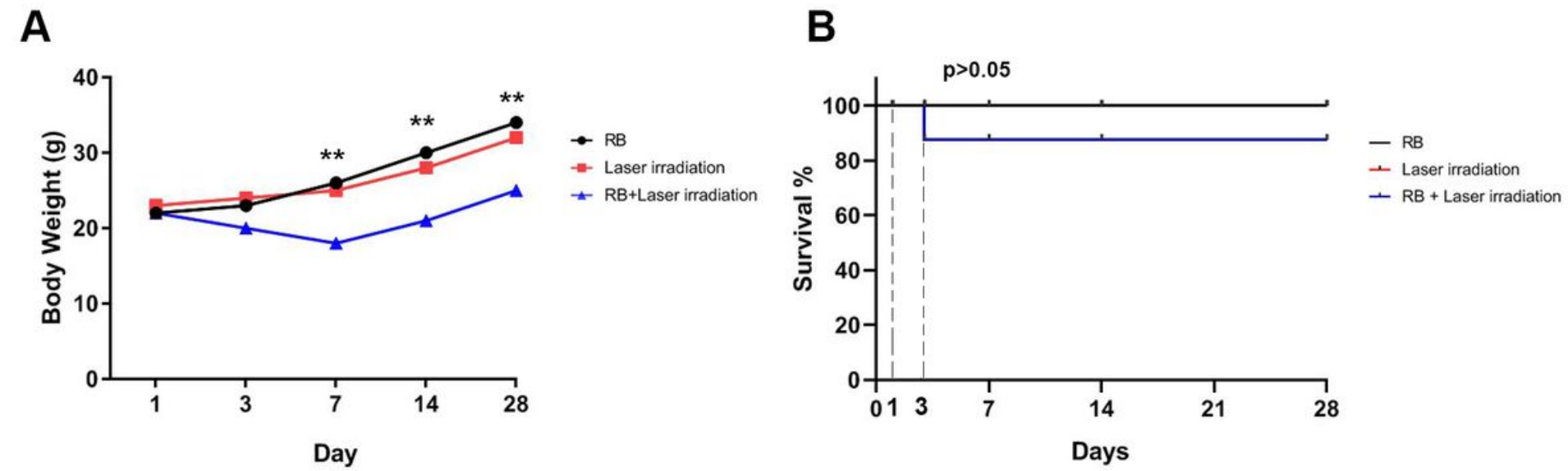

\section{Figure 2}

Monitoring body weight changes after PT-induced ischemic stroke (A); mortality rate evaluation by Kaplan Meier method (B). The body weight of mice decreased significantly during the first 7 days of stroke induction and then slowly increased and recovered to the baseline level after 14 days. The bodyweight of RB and laser irradiation groups did not alter. The total bodyweight of the RB + Laser irradiation group was $5 \%$ occurred during the early 3 days after PT-induced ischemic stroke which did not reach statistically significant levels compared to the Laser irradiation and RB groups $(p>0.05)(n=10)$. One-Way ANOVA and Tukey post hoc analysis. ${ }^{* \star} p<0.01$ 

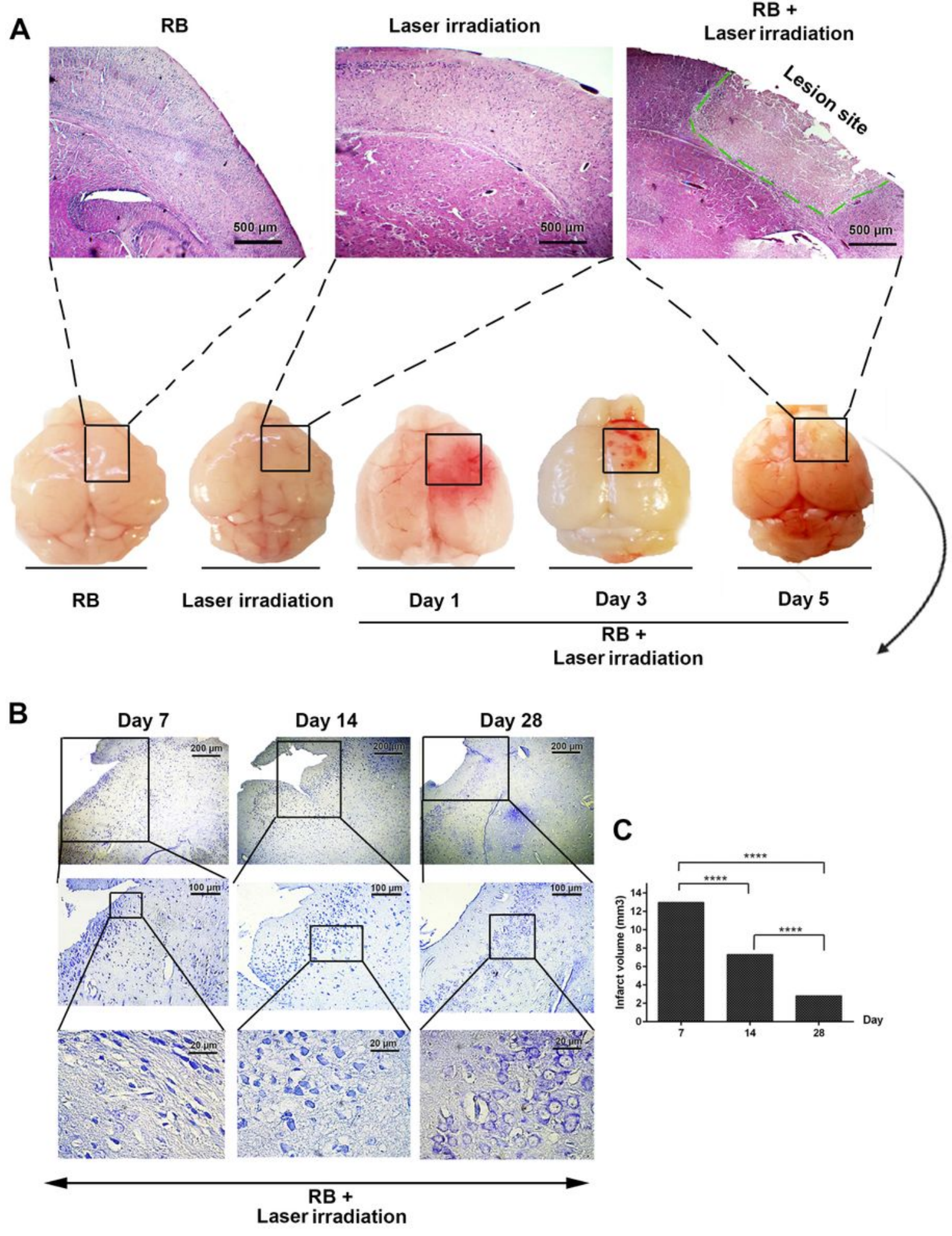

\section{Figure 3}

Macroscopic and microscopic evaluation of the brain after PT-induced ischemic stroke (A-C). H\& E staining of brain sections from RB, Laser irradiation, and RB + Laser irradiation groups (A). The macroscopic evaluation revealed disseminated hyperemia after 1 day, turned into ecchymosis hyperemia on day 3. The lesion sites were indicated with pale ischemic areas after 5 days. $\mathrm{H} \& \mathrm{E}$ staining revealed a necrotic area with delimited boundaries on 7 days. Monitoring tissue injury via Cresyl violet staining (B). 
Imaging revealed the massive neural injury and dense astrogliosis at the periphery of the lesion site, showing inflammatory response over time. The stereological analysis confirmed the decrease of lesion sites in RB + Laser irradiation groups over 28 days. One-Way ANOVA and Tukey post hoc analysis. $* \star * * p<0.0001$

A

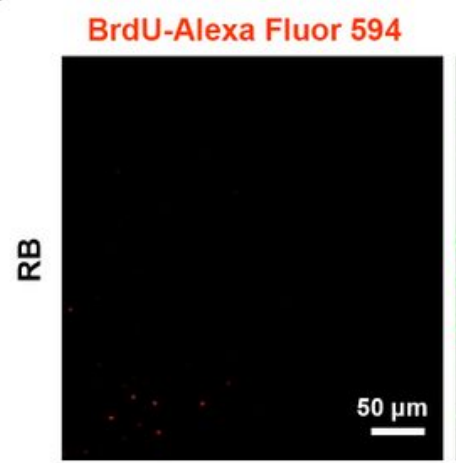

NeuN-Alexa Flour 488
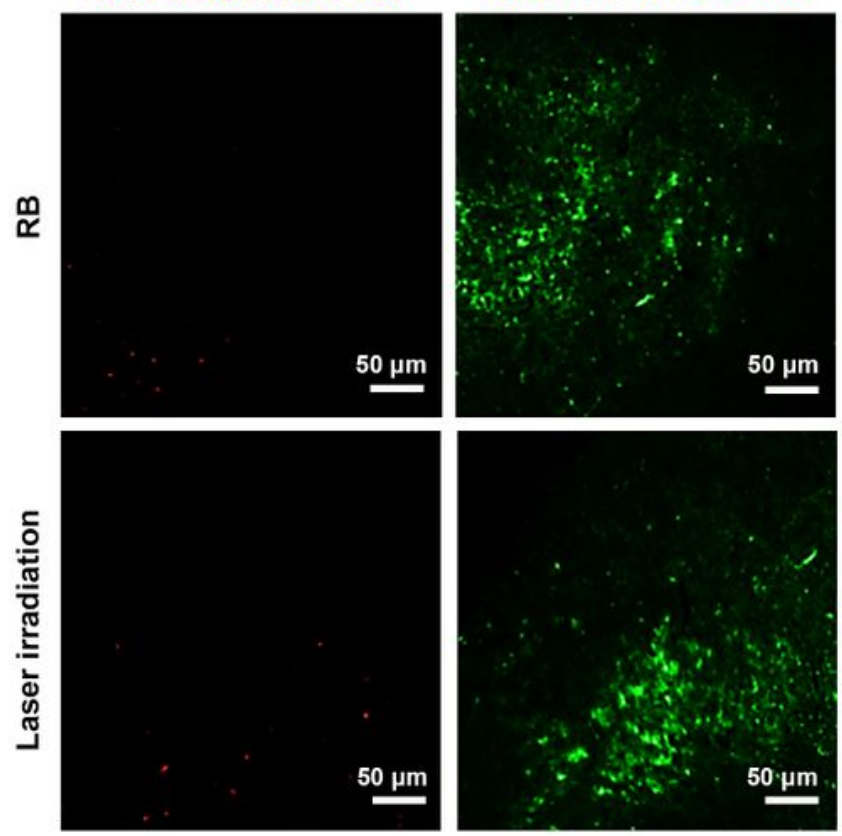

NeuN-Alexa Flour 488

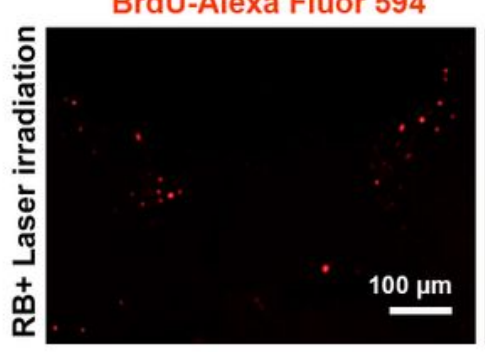

B

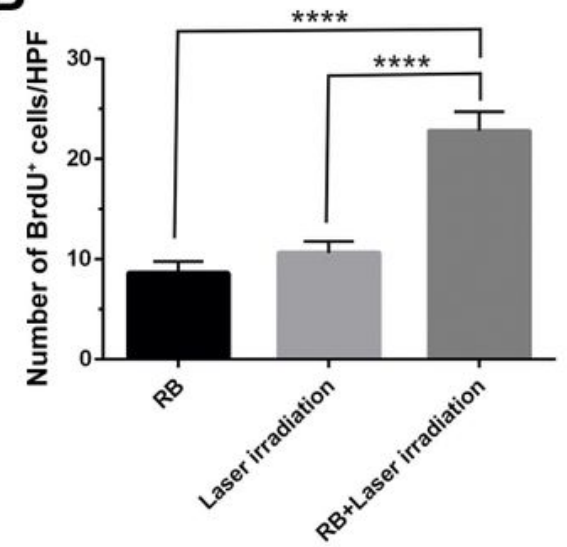

DAPI
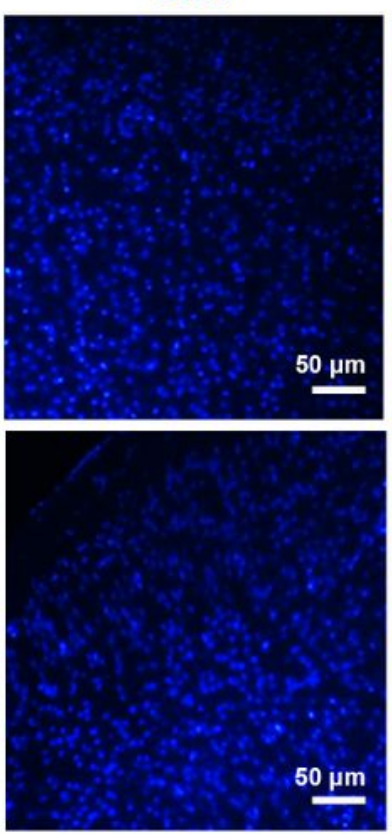

DAPI

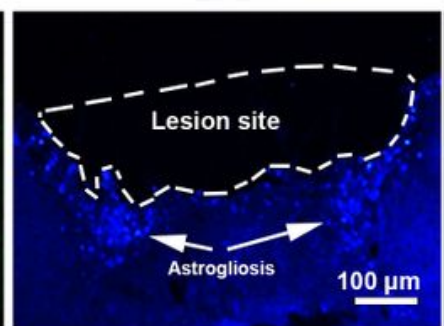

C

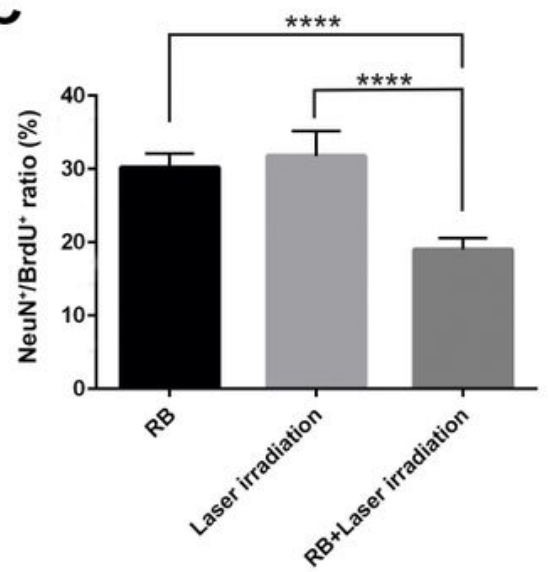

Figure 4 
Monitoring cell proliferation and neurogenesis ( $\mathrm{NeuN}+/ \mathrm{BrdU}+$ ratio) at the periphery site of the stroke area after 28 days $(A-C)$. The induction of stroke-induced cell proliferation at the periphery of lesion areas of the RB + Laser irradiation group compared to RB and Laser irradiation groups (B). According to our data, we found reduced $\mathrm{NeuN+/BrdU}+$ ratio in $\mathrm{RB}$ and Laser irradiation group compared to other groups. OneWay ANOVA and Tukey post hoc analysis. ${ }^{* * *} \mathrm{p}<0.0001$

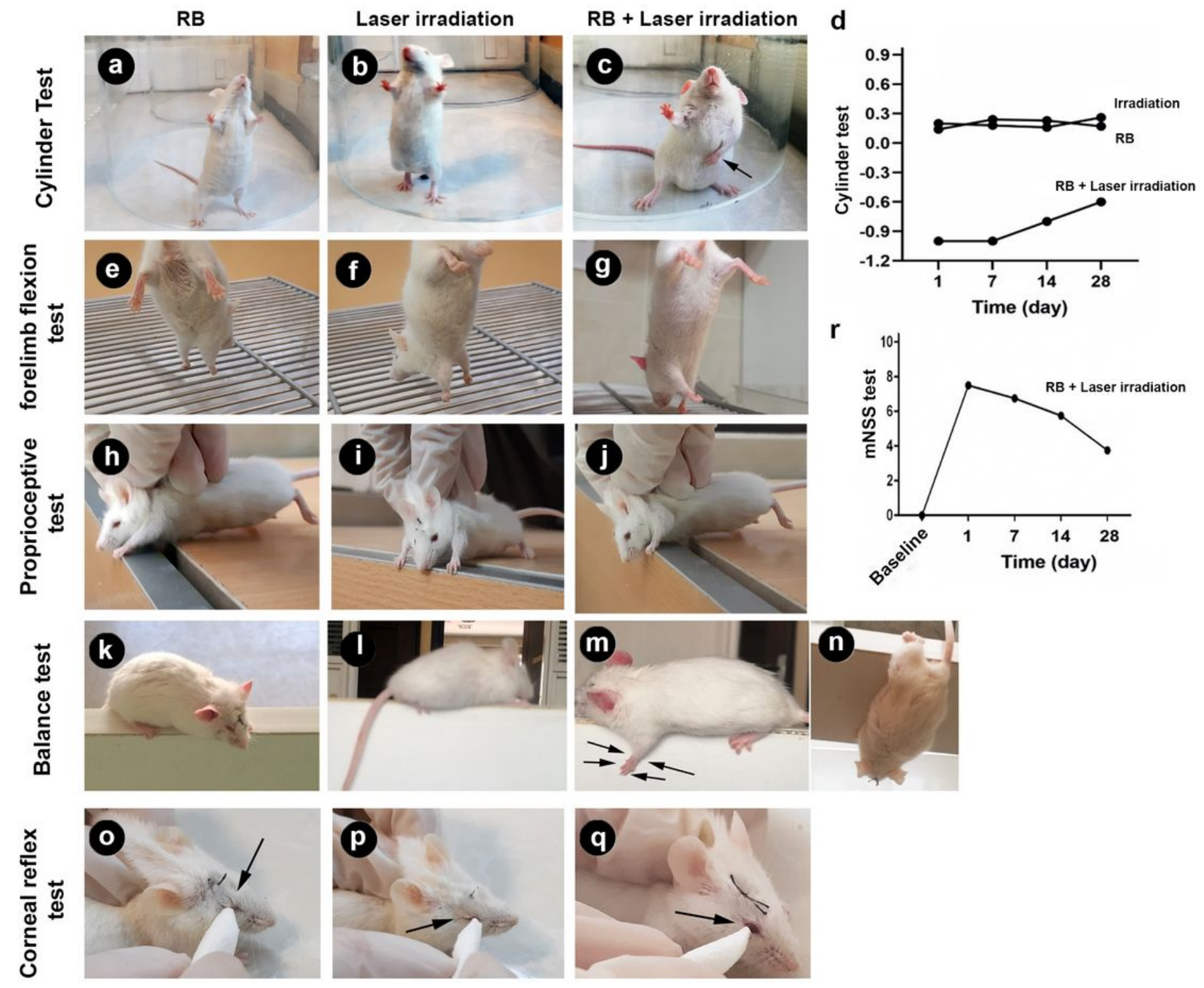

\section{Figure 5}

Neurological behavior and functional tests on days 1, 7, 14, 28 days after PT-induced ischemic stroke (ar). Cylinder test (a-d); Flexion forelimb test (e-g); Proprioceptive test (h-j); Balance test ( $k-n)$; Corneal reflex test (o-q)

\section{Supplementary Files}

This is a list of supplementary files associated with this preprint. Click to download. 
- 200AuthorChecklistFull.pdf

Page 24/24 